\title{
QCD Lambda parameter from Landau-gauge gluon and ghost correlations
}

\section{A. Sternbeck ${ }^{* a b}$, E.-M. Ilgenfritz ${ }^{c}$, K. Maltman ${ }^{a d}$, M. Müller-Preussker ${ }^{c}$,} L. von Smekal ${ }^{a e}$ and A. G. Williams ${ }^{a}$

${ }^{a}$ CSSM, School of Chemistry and Physics, University of Adelaide, SA 5005, Australia

${ }^{b}$ Institut für Theoretische Physik, Universität Regensburg, D-93040 Regensburg, Germany

${ }^{c}$ Humboldt-Universität zu Berlin, Institut für Physik, D-12489 Berlin, Germany

${ }^{d}$ Department of Mathematics and Statistics, York Univ., Toronto, ON, M3J 1P3, Canada

e Institut für Kernphysik, Technische Universität Darmstadt, D-64289 Darmstadt, Germany

We utilise a recently developed minimal MOM scheme to determine the QCD Lambda parameter from the gluon and ghost propagators in lattice Landau gauge. We discuss uncertainties in the analysis and report our preliminary zero and two flavour results, which are $r_{0} \Lambda \frac{(0)}{\mathrm{MS}}=0.62(1)$ and $r_{0} \Lambda \frac{(2)}{\mathrm{MS}}=0.60(3)(2)$, with the second error due to an extrapolation uncertainty.

The XXVII International Symposium on Lattice Field Theory - LAT2009

July 26-31, 2009

Peking University, Beijing, China

${ }^{*}$ Speaker. 


\section{Introduction}

The strong coupling constant $\alpha_{s}=g^{2} /(4 \pi)$ is one of the $N_{f}+1$ input parameters of QCD and as such one of the fundamental constants of nature. Its actual value depends on both the renormalisation scheme (including the number of active flavours) and the scale. Given a renormalisation scheme $\mathrm{S}$ the dependence on the scale $\mu$ is controlled by the renormalisation group (RG) through

$$
\mu^{2} \frac{d}{d \mu^{2}} \frac{\alpha_{s}^{\mathrm{S}}\left(\mu^{2}\right)}{\pi}=\beta^{\mathrm{S}}\left(\alpha_{s}^{\mathrm{S}}\right) \stackrel{\alpha_{s}^{\mathrm{S}} \rightarrow 0}{\sim}-\sum_{i \geq 0} \beta_{i}^{\mathrm{S}}\left(\frac{\alpha_{s}^{\mathrm{S}}}{\pi}\right)^{i+2},
$$

where $\beta^{S}$ is the beta function in that scheme. Solving Eq. (1.1) yields an exact relation between the scale-dependent coupling $\alpha_{s}^{\mathrm{S}}(\mu)$ and the RG-invariant, scale-independent but renormalisationscheme-dependent parameter $\Lambda_{\mathrm{S}}$, defined via $\ln \mu^{2} / \Lambda_{\mathrm{S}}^{2}=\int d \alpha_{s}^{\mathrm{S}} / \beta^{\mathrm{S}}(\alpha)$. Once the Lambda parameter is known for one scheme, a one-loop calculation suffices to determine it in any other scheme.

While recent precision determinations of $\alpha_{s}$ exist, based on either perturbative analyses of short-distance-sensitive lattice observables or sum rule analyses of hadronic $\tau$ decay data (for detailed discussions and other relevant references, see [1,2] and, e.g., [3], respectively), residual uncertainties mean that additional independent high-precision determinations remain of interest. Some of us have recently introduced the minimal momentum subtraction (MiniMOM, or MM) scheme for QCD in covariant gauges [4]. An important advantage of this scheme is that it allows the strong coupling to be fixed solely through a determination of the gluon and ghost propagators. In Landau gauge this scheme has been implicit in the early studies of these propagators [5].

The MM scheme is defined by combining MOM scheme propagator renormalisation with the supplementary condition $\widetilde{Z}_{1}=\widetilde{Z}_{1}^{\mathrm{MS}}$ for the ghost-gluon vertex renormalisation constant [4]. With $Z$ and $G$ the respective gluon and ghost dressing functions, the MM coupling is then defined as [5]

$$
\alpha_{s}^{\mathrm{MM}}\left(p^{2}\right)=\frac{g^{2}}{4 \pi} Z\left(p^{2}\right) G^{2}\left(p^{2}\right)
$$

The relation between $\alpha_{s}^{\mathrm{MM}}$ and $\alpha_{s}^{\overline{\mathrm{MS}}}$ is known to four loops [4]. Here we use $\alpha_{s}^{\mathrm{MM}}$ to determine $\Lambda \frac{\left(N_{f}\right)}{\mathrm{MS}}$ (in units of $\left.r_{0}\right)$ for $N_{f}=0,2$ from continuum extrapolations of the product of the bare lattice Landau gauge propagators, as first proposed in [6]. ${ }^{1}$ The absence of vertex measurements in the method allows for a significantly improved accuracy in the lattice estimate for $\alpha_{s}^{\mathrm{MM}}$.

\section{Numerical setup}

The results below were obtained on both $N_{f}=0$ and $2 S U(3)$ gauge field configurations. The quenched configurations were thermalised using the standard Wilson gauge action, with $\beta$ ranging from 6.0 to 8.5, applying standard update cycles of heatbath and micro-canonical over-relaxation steps. The unquenched gauge field configurations were provided by the QCDSF collaboration, who used the same gauge action supplemented by $N_{f}=2$ clover-improved Wilson fermions at various values of the hopping-parameter $\kappa$ (see Tab. 1 for further details). All gauge configurations

\footnotetext{
${ }^{1}$ Our 4-loop expansion for $\beta^{\mathrm{MM}}(\alpha)$ was not worked out until January 2008. While the 3-loop version of this expansion in fact differs somewhat from the 3-loop MOM $h$ scheme result used in [6], the difference is small.
} 
Table 1: Parameters of gauge configurations. $r_{0} / a$ values are from [8-10]; $a[\mathrm{fm}]$ is for $r_{0}=0.467 \mathrm{fm}$.

\begin{tabular}{cccc|rccccc}
\hline \hline$\beta$ & $N^{4}$ & \multicolumn{1}{c}{$r_{0} / a$} & $a[\mathrm{fm}]$ & \multicolumn{1}{c}{$\beta$} & $\kappa$ & $N_{s}^{3} \times N_{t}$ & $r_{0} / a$ & $a[\mathrm{fm}]$ & $a m_{0}$ \\
\hline 6.0 & $32^{4}$ & 5.3677 & 0.087 & 5.25 & 0.13575 & $24^{3} \times 48$ & $5.532(40)$ & 0.0844 & 0.01414 \\
6.2 & $32^{4}$ & 7.3829 & 0.060 & 5.25 & 0.13600 & $24^{3} \times 48$ & $5.732(64)$ & 0.0815 & 0.00737 \\
6.4 & $32^{4}$ & 9.7415 & 0.048 & 5.29 & 0.13590 & $24^{3} \times 48$ & $5.835(30)$ & 0.0800 & 0.01456 \\
6.6 & $32^{4}$ & 12.5955 & 0.037 & 5.29 & 0.13620 & $24^{3} \times 48$ & $6.083(26)$ & 0.0768 & 0.00646 \\
6.9 & $48^{4}$ & 18.6757 & 0.025 & 5.29 & 0.13632 & $32^{3} \times 64$ & $6.153(62)$ & 0.0759 & 0.00323 \\
7.2 & $64^{4}$ & 27.11 & 0.017 & 5.40 & 0.13610 & $24^{3} \times 48$ & $6.714(64)$ & 0.0696 & 0.01575 \\
7.5 & $64^{4}$ & 37.71 & 0.012 & 5.40 & 0.13640 & $32^{3} \times 64$ & $6.829(71)$ & 0.0690 & 0.00767 \\
8.5 & $48^{4}$ & 122.73 & 0.004 & 5.40 & 0.13660 & $32^{3} \times 64$ & $6.895(63)$ & 0.0681 & 0.00230 \\
\hline \hline
\end{tabular}

were fixed to lattice Landau gauge using an iterative gauge-fixing algorithm. To guarantee highprecision the local violation of transversality was not allowed to exceed $\varepsilon<10^{-10}$ where, as usual, $\varepsilon \equiv \max _{x} \mathfrak{R e} \operatorname{Tr}\left[\left(\nabla_{\mu} A_{x \mu}\right)\left(\nabla_{\mu} A_{x \mu}\right)^{\dagger}\right]$ and $\left.A_{x \mu} \equiv \frac{1}{2 i a g}\left(U_{x \mu}-U_{x \mu}^{\dagger}\right)\right|_{\text {traceless }}$.

Gluon and ghost propagators were measured on these gauge-fixed sets employing standard techniques and an acceleration for the Faddeev-Popov-operator inversion (see [6, 7] for details). As verified numerically in [7], for the range of momenta studied here the Gribov ambiguity is irrelevant. Using well established values for $r_{0} / a[8-10]$ to bring the raw data on $\alpha_{L}^{\mathrm{MM}}$ for different $\beta$ (and $\kappa$ ) onto the common scale $r_{0}^{2} p^{2}$ (see Tab. 1 for the $r_{0} / a$ values), and with $g^{2}(a)=6 / \beta(a)$ the bare coupling at the lattice cutoff scale $a^{-1}, \alpha_{s}^{\mathrm{MM}}$ was then determined from the averaged data for the bare lattice gluon and ghost propagator dressing functions, $Z_{L}$ and $G_{L}$, via

$$
\alpha_{s}^{\mathrm{MM}}\left(p^{2}\right)=\alpha_{L}^{\mathrm{MM}}\left(p^{2}\right)+O\left(a^{2}\right) \quad \text { with } \quad \alpha_{L}^{\mathrm{MM}}\left(p^{2}\right) \equiv \frac{g^{2}(a)}{4 \pi} Z_{L}\left(p^{2}, a^{2}\right) G_{L}^{2}\left(p^{2}, a^{2}\right) .
$$

To have the lattice tree-level structure correct the dressing functions were extracted using $a q_{\mu}\left(p_{\mu}\right)=$ $2 \sin \left(a p_{\mu} / 2\right)$, but $\alpha_{s}^{\mathrm{MM}}$ is considered versus $p^{2}$ with $a p_{\mu}=2 \pi k_{\mu} / L_{\mu}$ and $k_{\mu} \in\left(-L_{\mu} / 2, L_{\mu} / 2\right]$.

\section{Lattice data of the MiniMOM coupling}

Our lattice data for $\alpha_{L}^{\mathrm{MM}}\left(p^{2}\right)$ is shown in Fig. 1, together with the expected 4-loop continuum running obtained using, to be specific, the ALPHA collaboration $\overline{\mathrm{MS}}$ values, $r_{0} \Lambda_{\overline{\mathrm{MS}}}^{(0)}=0.60(5)$, and $r_{0} \Lambda \frac{(2)}{\mathrm{MS}}=0.62(4)(4)[11,12]$, translated to the MiniMOM scheme (see Table 4 of Ref. [4] for the relevant values of $\Lambda_{\overline{\mathrm{MS}}} / \Lambda_{\mathrm{MM}}$ ). In what follows, for the sake of illustration, we display momenta in physical units (rather than as $r_{0}^{2} p^{2}$ ), using $r_{0}=0.467 \mathrm{fm}=2.367 \mathrm{GeV}^{-1}$. Since only ratios of momenta enter the calculation, this choice does not affect the final result for $r_{0} \Lambda \frac{\left(N_{f}\right)}{\mathrm{MS}}$.

From Fig. 1, one sees that, for both $N_{f}=0,2$, scaling violations, finite volume effects and hypercubic lattice artefacts are nearly negligible, even though, for $N_{f}=0$, the lattice spacing varies over an order of magnitude. Small systematic deviations from continuum 4-loop running, however, become visible at higher resolution. Such deviations are negligible for purely diagonal lattice momenta satisfying $3<a^{2} p^{2}<6$, but grow, $\propto 1 /(a p)^{2}\left(\propto a^{2} p^{2}\right)$ to leading order, for momenta below (above) this interval. Fig. 1 shows data for diagonal momenta with $1<a^{2} p^{2}<10$.

Deviations from continuum 4-loop running are not unexpected. At small momenta, they result from a mixture of (a) finite volume effects, (b) the onset of nonperturbative effects (condensates etc.) and (c) truncation errors in the perturbative expansion of the coupling, while at large momenta 

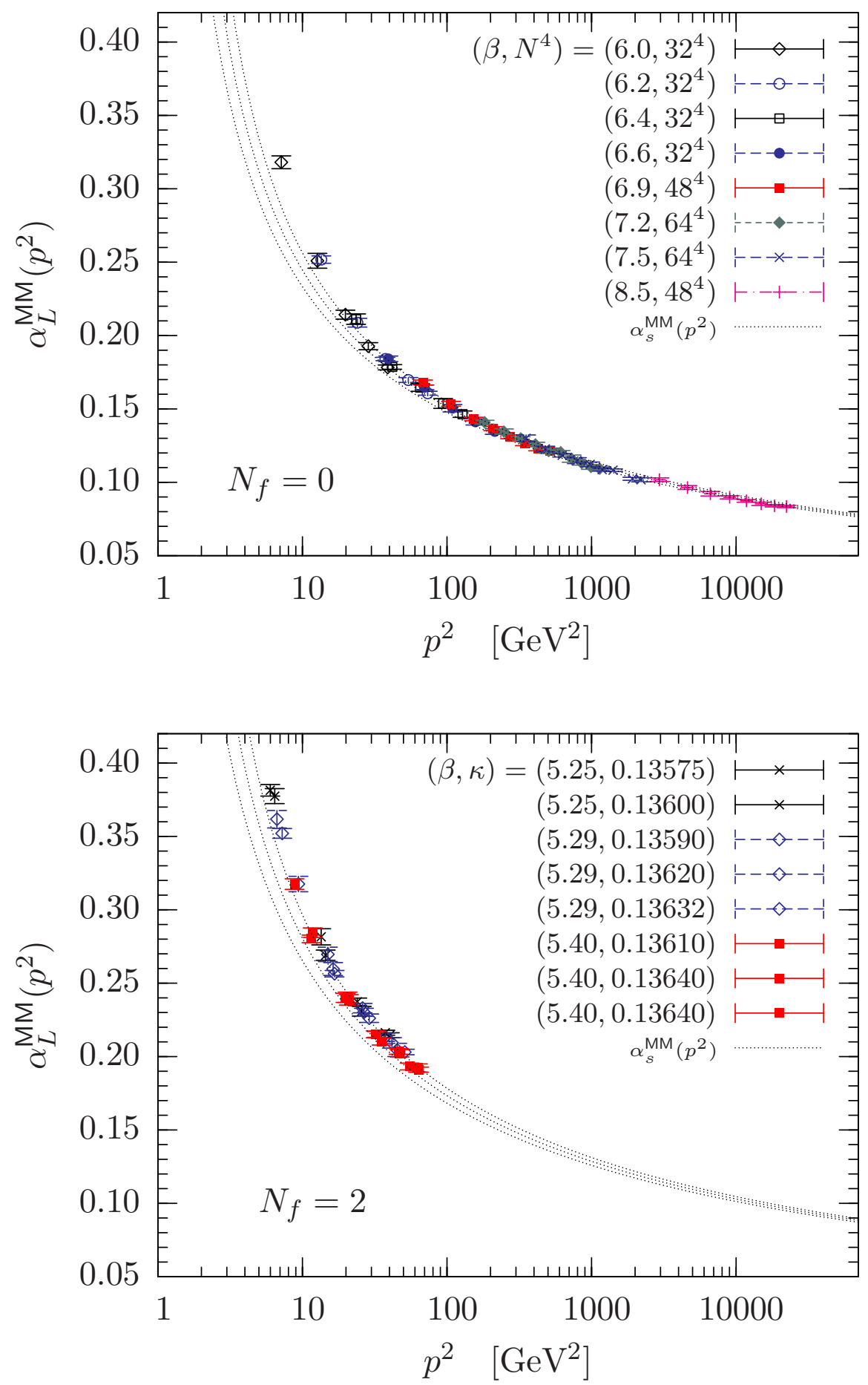

Figure 1: Lattice data for $\alpha_{s}^{\mathrm{MM}}\left(p^{2}\right)$ for $N_{f}=0$ (top) and $N_{f}=2$ (bottom) compared to the expected 4-loop running of $\alpha_{s}^{\mathrm{MM}}\left(p^{2}\right)$ (lines) in the continuum limit [4]. Note that these are not fits: The data is the raw lattice data for purely diagonal momenta $a p_{\mu}=2 \pi k_{\mu} / L_{\mu}$ with $1<a^{2} p^{2}<10$ brought onto a common scale employing established values for $r_{0} / a$ for these sets [8-10]. For illustration purposes, the 4-loop running has been fixed using $r_{0} \Lambda \frac{(0)}{\mathrm{MS}}=0.60(5)$ and $r_{0} \Lambda \frac{(2)}{\mathrm{MS}}=0.62(4)(4)$ of the ALPHA collaboration [11, 12], and the overall momentum scale set using $r_{0}=0.467 \mathrm{fm}=2.367 \mathrm{GeV}^{-1}$. 
they are due to (d) scaling violations proportional to $a^{2} p^{2}$ and (e) hypercubic lattice artefacts proportional to higher-order invariants $a^{n} p^{[n]}=\sum_{\mu} a^{n} p_{\mu}^{n}(n=4,6,8)$ of the isometry group $H(4)$. The latter, in particular, would become pronounced for larger momenta without suitable corrections, which can be performed either using the $\mathrm{H} 4$ method or by imposing so-called cylinder cuts on the data (for the former approach, see, e.g., [13], for the latter [14]). The cylinder cut approach, though less sophisticated, is nonetheless effective and robust. We chose a combination of the two methods to keep the statistical noise to a minimum. To be specific, we consider data only for purely diagonal lattice momenta, for which hypercubic lattice artefacts are known to be smallest, and correct for the remaining (rather small) artefacts through a fit of this data to a hypercubic Taylor expansion of the (lattice) MM coupling which, to leading order, has the form

$$
\alpha_{L}^{\mathrm{MM}}\left(p^{2}\right)=\alpha_{s}^{\mathrm{MM}}\left(p^{2}\right)\left(1+c_{1} \cdot a^{2} p^{2}+c_{2} \cdot a^{4} p^{4}+\ldots\right)
$$

where the $c_{i}$ are constants (see also $[13,15,16]$ ). For the classic $\mathrm{H} 4$ method one would have to fit the $c_{i}$ 's from extrapolations of the $\alpha_{L}^{\mathrm{MM}}\left(p^{2}\right)$ data at different lattice momenta belonging to different $H(4)$ orbits but the same $a^{2} p^{2}$. Not only are these extrapolations susceptible to statistical artefacts for insufficiently large lattice sizes, but the Faddeev-Popov operator has to be inverted using point sources to get data for all momenta, introducing larger statistical fluctuations into the ghost propagator (and hence the coupling) at large momenta. Our approach allows us to use instead plane-wave sources for our inversions (see [7] for details) and fully exploits the translation invariance of the lattice, thus drastically reducing statistical noise in the coupling. The results below

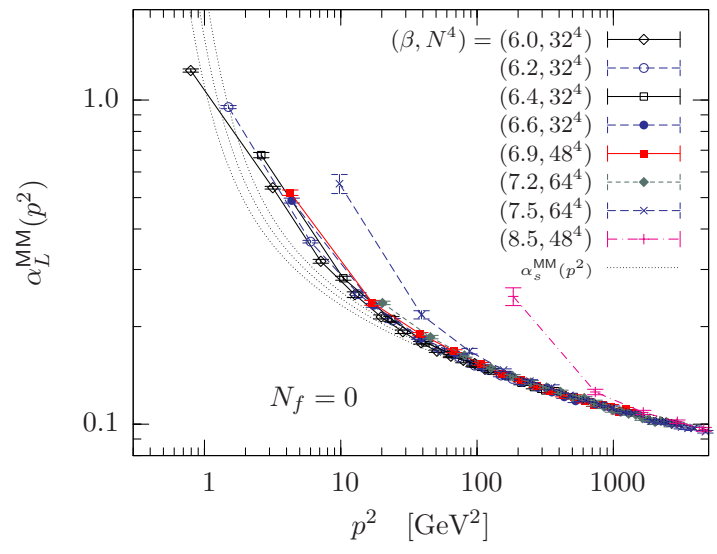

Figure 2: $N_{f}=0$ data for $\alpha_{L}^{\mathrm{MM}}\left(p^{2}\right)$ as shown in Fig. 1 but here for $0<a^{2} p^{2}<23$ to illustrate finite volume effects at small momenta which are more pronounced here than the lattice artefacts at large. bear out the reasonableness of the approach, providing an excellent description of the data for $3<a^{2} p^{2}<30$ (or $3<a^{2} p^{2}<12$ for $c_{2} \equiv 0$ ) and giving stable results for all fit parameters.

The deviations at small momenta, illustrated in Fig. 2, are more severe and, in our opinion, not yet fully under control. They start to become visible for $a^{2} p^{2}<1$ and appear to be a mixture of finite volume and nonperturbative effects (the latter expected to set in at smaller $\beta$ ). The effect is such that data points at fixed physical momenta decrease as the physical volume increases. Currently, additional simulations at different $\beta$ but fixed physical volume, are being performed to help bring these low-momentum artefacts under better control. For now, we exclude data with $a^{2} p^{2}<3$ to stay well clear of the region where such effects become evident.

\section{Fitting the data}

Our fitting procedure works as follows. Each of the data sets is fitted separately to the Ansatz (3.1), where the 4-loop perturbative running form is used for $\alpha_{s}^{\mathrm{MM}}\left(p^{2}\right)$ and the remaining terms correct for the leading lattice artefacts at larger $a^{2} p^{2}$. All fits are performed using the 

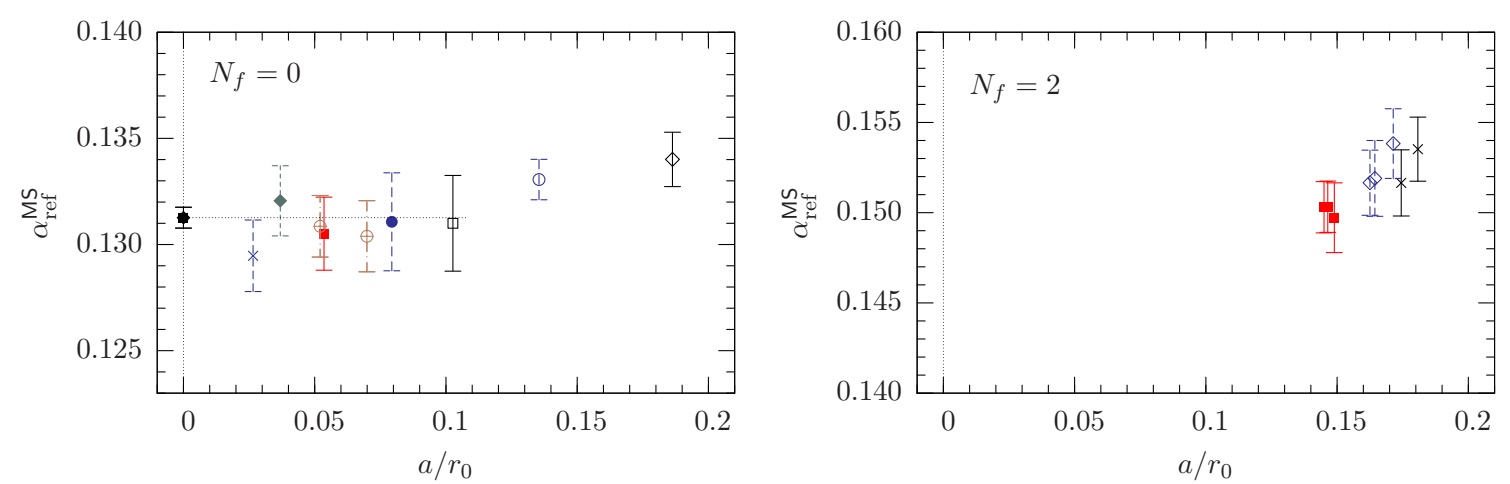

Figure 3: Values for $\alpha_{\mathrm{ref}}^{\overline{\mathrm{MS}}}$ obtained from fits to the data for different $a / r_{0}$ using Ansatz (3.1); left for $N_{f}=0$ and right for $N_{f}=2$ and different bare quark masses $a_{0}$. See text and Tab. 1 for further details. Symbols refer to the same $\beta, N^{4}$ (and $\kappa$ ) as in Fig. 1. The dotted line and very left 'star' (left panel) are a fit of the plateau of the three points (filled symbols), calculated in equal physical volumes $V_{1} \approx(1.2 \mathrm{fm})^{4}$. Note that there we have also included two very recent points (brown crossed circles) from simulations at $\beta=6.7$ and 6.92 and equal physical volume, $V_{2} \approx(1.6 \mathrm{fm})^{4}$.

fixed fitting window $3<a^{2} p^{2}<12$. (The fits have been checked to be quite robust to small changes to the lower and upper bounds of this window.) We also find that $c_{2}$ can be set to 0 with little effect on $c_{1}$. The 4-loop running of $\alpha_{s}^{\mathrm{MM}}\left(p^{2}\right)$ is generated from that of $\alpha_{s}^{\overline{\mathrm{MS}}}\left(p^{2}\right)$ using the 4-loop relation between the couplings given in Eqs. (14-15) of Ref. [4]. (Further, more specific details will be provided in an upcoming publication.)

With $c_{2}$ set to zero, our fit parameters reduce to $c_{1}$, the constant of the leading lattice correction at larger momenta, and $\alpha_{\mathrm{ref}}^{\overline{\mathrm{MS}}}$, the $\overline{\mathrm{MS}}$ coupling at an arbitrary reference scale $p_{\text {ref }}^{2}$ (to be specific, we take $p_{\text {ref }}^{2}=70 \mathrm{GeV}^{2}$ and $\left.r_{0}=0.467 \mathrm{fm}\right) . \Lambda_{\overline{\mathrm{MS}}}$ could, of course, be used in place of $\alpha_{\text {ref }}^{\overline{\mathrm{MS}}}$ as a fit parameter; we expect our fits to be more stable with the latter choice. $\Lambda_{\overline{\mathrm{MS}}}$ in any case follows from $\alpha_{\text {ref }}^{\overline{\mathrm{MS}}}$ using the standard relation [17]

$$
\ln \frac{\mu^{2}}{\Lambda^{2}}=\int \frac{d a}{\beta(a)}=\frac{1}{\beta_{0}}\left[\frac{1}{a}+b_{1} \ln a+\left(b_{2}-b_{1}^{2}\right) a+\left(\frac{b_{3}}{2}-b_{1} b_{2}+\frac{b_{1}^{3}}{2}\right) a^{2}\right]+C
$$

where $a(\mu) \equiv \alpha_{s}(\mu) / \pi, \beta_{0}^{\overline{\mathrm{MS}}}, \ldots, \beta_{3}^{\overline{\mathrm{MS}}}$ are the $\overline{\mathrm{MS}}$ scheme $\beta$-function coefficients, $b_{i}=\beta_{i} / \beta_{0}$ and $C=\left(b_{1} / \beta_{0}\right) \ln \beta_{0}$.

Fitted values for $\alpha_{\mathrm{ref}}^{\overline{\mathrm{MS}}}$ and $c_{1}$ as a function of $a / r_{0}$ (and, for $N_{f}=2$, also for different bare quark masses) are shown in Figs. 3 and 4. Note that if the data showed perfect scaling, all fits would give the same value (within errors) for $\alpha_{\mathrm{ref}}^{\overline{\mathrm{MS}}}$, independent of the lattice spacing, and the fitted $O\left(a^{2}\right)$ corrections $\left(c_{1}\right)$ would turn out to be zero. As expected, $c_{1}$, though small, is not zero (see Fig. 4). The long plateau for $c_{1}$ as a function of $a / r_{0}$, however, suggests that our Ansatz for describing the leading lattice corrections is a reliable one. This conclusion is also supported by the results for $\alpha_{\mathrm{ref}}^{\overline{\mathrm{MS}}}$. For $N_{f}=0$, for example, $\alpha_{\mathrm{ref}}^{\overline{\mathrm{MS}}}$ starts to plateau around $a / r_{0}=0.1$, i.e., for $\beta \geq 6.4$ (see Fig. 3). Note that the fit quality is significantly degraded if no correction term is included.

For $N_{f}=2$ we observe only small deviations in $\alpha_{\mathrm{ref}}^{\overline{\mathrm{MS}}}$ on changing the bare quark mass (see Fig. 3, right panel). Changing $a / r_{0}$ leads to more significant shifts. This is almost certainly due to the relatively large $a / r_{0}$ employed, and the fact that the lattice data available for $\alpha_{s}^{\mathrm{MM}}$ come 

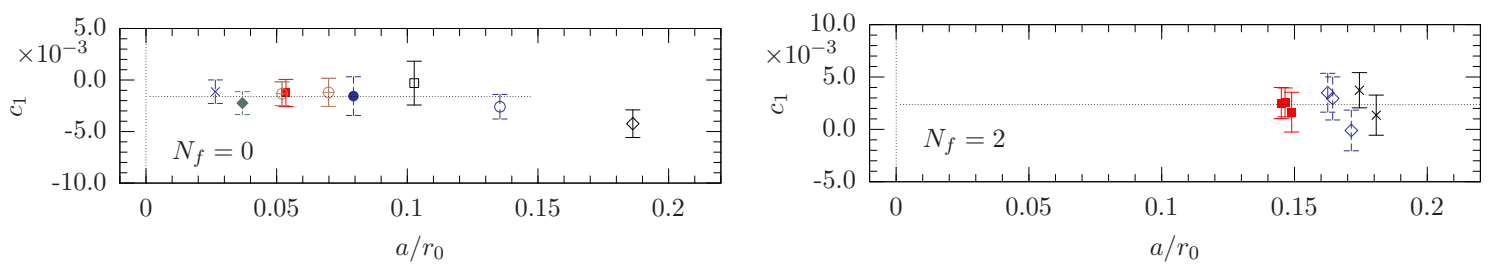

Figure 4: Coefficient $c_{1}$ of the leading lattice correction (see Eq. (3.1)) for different $a / r_{0}$, obtained from the same fits as $\alpha_{\text {ref }}^{\overline{\mathrm{MS}}}$ shown in Fig. 3. Symbols are the same as in Figs. 1 and 3. Dotted lines mark the average $c_{1}$ including data left for $a / r_{0}<0.15$ and right for $a / r_{0}<0.2$.

from momenta where deviations from the 4-loop running of $\alpha_{s}^{\mathrm{MM}}$ have already set in (see Fig. 1, bottom panel). The overall picture, however, appears similar to that for $N_{f}=0$, and the fitted $\alpha_{\mathrm{ref}}^{\overline{\mathrm{MS}}}$ for the $\beta=5.40$ configurations are already close to what is expected, for example, from [12]. In fact, $r_{0} \Lambda \frac{(2)}{\mathrm{MS}}=0.62(4)(4)$ corresponds to $\alpha_{\mathrm{ref}}^{\overline{\mathrm{MS}}}=0.150(3)$ in the right panel of Fig. 3.

A continuum extrapolation in our approach corresponds to fitting data plateaus visible at small enough $a / r_{0}$. While it is far from clear that a plateau has been reached for the $N_{f}=2$ data in Fig. 3 the $N_{f}=0 \alpha_{\mathrm{ref}}^{\overline{\mathrm{MS}}}$ results level off quite nicely at smaller $a / r_{0}$. Fitting the three points having the same physical volume, i.e., those with $(\beta, N)=\{(6.6,32),(6.9,48),(7.2,64)\}$, yields $\alpha_{\text {ref }}^{\overline{\mathrm{MS}}}=0.131(1)$. This corresponds to $r_{0} \Lambda \frac{(0)}{\mathrm{MS}}=0.62(1)$ which agrees well with values from the literature. Assuming that the relative decrease of $\alpha_{\mathrm{ref}}^{\overline{\mathrm{MS}}}$ from $a / r_{0}=0.15$ to $\sim 0.1$ will be the same for $N_{f}=0$ and 2 (about $1.5 \%$ ), one would expect the $N_{f}=2$ data to level off at smaller $a / r_{0}$ around $\alpha_{\text {ref }}^{\overline{\mathrm{MS}}}=0.148(2)$. This would correspond to $r_{0} \Lambda \frac{(2)}{\mathrm{MS}}=0.59(3)$, also in good agreement with existing values in the literature. Additional data for $N_{f}=2$ at smaller $a / r_{0}$ would allow us to make further progress, but adequate gauge configurations are unfortunately not yet available. For now we take the average $r_{0} \Lambda \frac{(2)}{\mathrm{MS}}=0.60(3)(2)$ with the second error due to the uncertainty in the continuum extrapolation.

\section{Conclusions}

In this paper, we have taken advantage of the recently introduced MM scheme for QCD in covariant gauges [4] to perform a determination of the QCD Lambda parameter for $N_{f}=0,2$. The scheme allows the strong coupling constant, and hence $\Lambda_{\overline{\mathrm{MS}}}$, to be determined from measurements of ghost and gluon two-point functions on the lattice. The restriction to measured two-point functions, and the fact that the relation between the $\mathrm{MM}$ and $\overline{\mathrm{MS}}$ couplings is known to 4-loop order, allows for a high precision determination, with reliable error estimates.

Our results to date are restricted to $N_{f}=0,2$, and must be extended to $N_{f}=2+1$ in order to reach the desired goal of estimating $\alpha_{s}^{\overline{\mathrm{MS}}}\left(M_{Z}\right)$. Fortunately, over the last few years, the number of available $N_{f}=2+1$ gauge configurations has increased significantly, and continues to increase. The $N_{f}=0,2$ results presented above, which yield the (still preliminary) results

$$
r_{0} \Lambda \frac{(0)}{\mathrm{MS}}=0.62(1) \quad \text { and } \quad r_{0} \Lambda \frac{(2)}{\mathrm{MS}}=0.60(3)(2),
$$

in agreement with other studies (e.g., $[10-12,16])$, demonstrate the reliability and accuracy of our method, and thus pave the way for future $N_{f}=2+1$ analyses. The analysis also provides valuable 
information on how to bring lattice artefacts under control.

A positive feature of the current study is that lattice artefacts are found to be almost negligible if one restricts the analysis to strictly diagonal lattice momenta satisfying $3<a^{2} p^{2}<6$. For larger momenta, hypercubic lattice artefacts become visible. For $a^{2} p^{2}<12$ they grow like $c_{1} \alpha_{s}^{\mathrm{MM}}\left(p^{2}\right) a^{2} p^{2}$, with $c_{1}=-0.00016(3)$ for $N_{f}=0$, and $c_{1}=0.00023(4)$ for $N_{f}=2$, and thus can be corrected for quite precisely. It is anticipated that the precision could be improved further if data from lattice perturbation theory was available (see, e.g, [18] for steps in this direction).

For smaller momenta, finite volume effects are present, in particular for $a^{2} p^{2}<1$. These effects are not yet fully under control and new calculations at different $\beta$ but fixed volumes are under way to rectify this situation. It is for this reason that we have not yet investigated condensate effects, which are expected to be relevant for lower momenta (see, for example, [16]).

Note that the MM coupling could also be employed to determine the lattice spacing dependence $a(\beta)$ via $a \Lambda_{\overline{\mathrm{MS}}}(\beta)$. With this information, and the ratios of the $\Lambda_{\overline{\mathrm{MS}}}^{\left(N_{f}\right)}$ for $N_{f}=0,2$ and 3 , a well-established experimental value for $\alpha_{s}^{\overline{\mathrm{MS}}}\left(M_{Z}\right)$ could be used to fix the physical scale of $a$ for different $N_{f}$ (rather than using $r_{0}$ ). This again would require a good understanding of all lattice artefacts since $\alpha_{s}^{\mathrm{MM}}$ data for different lattice spacings would have to be brought to a common scale via a matching procedure, starting in the perturbative region.

This research was supported by the Australian Research Council. A.S. is also supported by the $\mathrm{Sfb} / \mathrm{Tr}-55$ K.M. by the Natural Sciences and Engineering Council of Canada, and L.v.S by the Helmholtz International Center for FAIR within the LOEWE program of the State of Hessen, Germany. K.M. also acknowledges the hospitality of the CSSM at the University of Adelaide. Grants of time on the computing facilities of the HLRN-Verbund (Germany) and eResearchSA (Australia) are acknowledged.

\section{References}

[1] C. T. H. Davies et al. Phys. Rev. D78 (2008) 114507.

[2] K. Maltman, D. Leinweber, P. Moran, and A. Sternbeck Phys. Rev. D78 (2008) 114504.

[3] K. Maltman and T. Yavin Phys. Rev. D78 (2008) 094020.

[4] L. von Smekal, K. Maltman, and A. Sternbeck Phys. Lett. B681 (2009) 336.

[5] L. von Smekal, R. Alkofer, and A. Hauck Phys. Rev. Lett. 79 (1997) 3591; Ann. Phys. 267 (1998) 1.

[6] A. Sternbeck et al. PoS LAT2007 (2007) 256, [0710 . 2965].

[7] A. Sternbeck, E. M. Ilgenfritz, M. Müller-Preussker, and A. Schiller Phys. Rev. D72 (2005) 014507.

[8] S. Necco and R. Sommer Nucl. Phys. B622 (2002) 328.

[9] M. Guagnelli, R. Petronzio, and N. Tantalo Phys. Lett. B548 (2002) 58.

[10] M. Göckeler et al. Phys. Rev. D73 (2006) 014513; Database of the QCDSF collaboration, Feb. 2010.

[11] S. Capitani, M. Lüscher, R. Sommer, and H. Wittig Nucl. Phys. B544 (1999) 669.

[12] M. Della Morte et al. Nucl. Phys. B713 (2005) 378.

[13] F. de Soto and C. Roiesnel JHEP 09 (2007) 007.

[14] D. B. Leinweber, J. I. Skullerud, A. G. Williams, and C. Parrinello Phys. Rev. D60 (1999) 094507.

[15] F. Di Renzo, V. Miccio, L. Scorzato, and C. Torrero Eur. Phys. J. C51 (2007) 645.

[16] P. Boucaud et al. Phys. Rev. D79 (2009) 014508;

F. De soto, M. Gravina, O. Pene, and J. Rodriguez-Quintero, 0911.4505

[17] K. G. Chetyrkin, B. A. Kniehl, and M. Steinhauser Phys. Rev. Lett. 79 (1997) 353.

[18] F. Di Renzo, E. M. Ilgenfritz, H. Perlt, A. Schiller, and C. Torrero Nucl. Phys. $\mathbf{B 8 3 1}$ (2010) 262; F. Di Renzo, E. M. Ilgenfritz, H. Perlt, A. Schiller, and C. Torrero, 0910.2905. 